\title{
Reduced Mortality and Severe Disability Rates in the SENTIS Trial
}

P.D. Schellinger, A. Shuaib, M. Köhrmann, D.S. Liebeskind, T. Jovin, M.D. Hammer, S. Sen, D.Y. Huang, S. Solander, R. Gupta, R.R. Leker, and J.L. Saver, for the SENTIS Trial Investigators

O-. EBM

\begin{abstract}
BACKGROUND AND PURPOSE: The Safety and Efficacy of NeuroFlo Technology in Ischemic Stroke trial showed a trend for reduced all-cause mortality and positive secondary safety end point outcomes. We present further analyses of the mortality and severe disability data from the Safety and Efficacy of NeuroFlo Technology in Ischemic Stroke trial.

MATERIALS AND METHODS: The Safety and Efficacy of NeuroFlo Technology in Ischemic Stroke trial was a multicenter, randomized, controlled trial that evaluated the safety and effectiveness of the NeuroFlo catheter in patients with stroke. The current analysis was performed on the as-treated population. All-cause and stroke-related mortality rates at 90 days were compared between groups, and logistic regression models were fit to obtain ORs and $95 \% \mathrm{Cls}$ for the treated versus not-treated groups. We categorized death-associated serious adverse events as neurologic versus non-neurologic events and performed multiple logistic regression analyses. We analyzed severe disability and mortality by outcomes of the mRS. Patient allocation was gathered by use of a poststudy survey.
\end{abstract}

RESULTS: All-cause mortality trended in favor of treated patients (11.5\% versus $16.1 \% ; P=.079)$ and stroke-related mortality was significantly reduced in treated patients $(7.5 \%$ versus $14.2 \% ; P=.009)$. Logistic regression analysis for freedom from stroke-related mortality favored treatment (OR, 2.41; 95\% Cl, 1.22, 4.77; $P=.012)$. Treated patients had numerically fewer neurologic causes of stroke-related deaths (52.9\% versus $73.0 \% ; P=.214$ ). Among the 90 -day survivors, nominally fewer treated patients were severely disabled (mRS 5) (5.6\% versus $7.5 \%$; OR, 1.72; $95 \% \mathrm{Cl}, 0.72,4.14 ; P=.223)$. Differences in allocation of care did not account for the reduced mortality rates.

CONCLUSIONS: There were consistent reductions in all-cause and stroke-related mortality in the NeuroFlo-treated patients. This reduction in mortality did not result in an increase in severe disability.

ABBREVIATIONS: SENTIS = Safety and Efficacy of NeuroFlo Technology in Ischemic Stroke; NIHSSS = National Institutes of Health Stroke Scale Score

$\mathbf{R}_{\mathrm{N}}^{\mathrm{c}}$ ecently, the primary results of the Safety and Efficacy of NeuroFlo Technology in Ischemic Stroke (SENTIS) trial have been published. ${ }^{1}$ NeuroFlo therapy involves partial occlusion of

Received January 8, 2013; accepted after revision March 21.

From JW Klinikum (P.D.S.), Minden, Germany; University of Alberta (A.S.), Edmonton, Alberta, Canada; Universitätsklinikum Erlangen (M.K.), Erlangen, Germany; University of California, Los Angeles (D.S.L., J.L.S.), Los Angeles, California; University of Pittsburgh Medical Center (T.J., M.D.H.), Pittsburgh, Pennsylvania; St Thomas Hospital (S.Sen), Nashville, Tennessee; University of South Carolina (D.Y.H.), Columbia, South Carolina; University of North Carolina (S.Solander), Chapel Hill, North Carolina; Emory University (R.G.), Atlanta, Georgia; and Hadassah Hebrew University Medical Center (R.R.L.), Jerusalem, Israel.

Clinical Trial Registration Information: URL: http://clinicaltrials.gov. Unique identifier: NCT00119717.

Please address correspondence to Peter D. Schellinger, MD, PhD, FESO, Johannes Wesling Klinikum Minden, Departments of Neurology and Neurogeriatry, HansNolte-Str 1, D-32429 Minden, Germany; e-mail: peter.schellinger@ muehlenkreiskliniken.de

- Indicates open access to non-subscribers at www.ajnr.org

Evidence-Based Medicine Level 1.

http://dx.doi.org/10.3174/ajnr.A3613 the abdominal aorta that results in a prompt increase in blood volume above the partial occlusion and has been shown to specifically increase CBF by diverting blood flow from the lower limbs to the brain. ${ }^{2}$ Preclinical studies in animal models and imaging studies in patients indicate that this intervention creates a substantial increase in CBF that persists beyond deflation and removal of the NeuroFlo device without an increase in intracerebral hemorrhages. ${ }^{1,3}$ A feasibility study in 25 patients with ischemic stroke treated up to 24 hours after symptom onset also showed no parenchymal hematomas and no symptomatic intracerebral hemorrhages. ${ }^{4}$ For an extensive review of the literature and mode of action, we refer to a recent review by Liebeskind et al. ${ }^{5}$

SENTIS was a prospective, randomized, multicenter trial to evaluate the safety and efficacy of CBF augmentation in patients with acute ischemic stroke who were able to have NeuroFlo treatment initiated within 14 hours of the onset of symptoms. SENTIS was the first randomized trial of an interventional device for stroke with a primary clinical outcomes end point, a global dis- 
ability outcome assessment that is based on an excellent outcome on a combination of several scales (Barthel Index, National Institutes of Health Stroke Scale Score [NIHSSS], mRS, and Glasgow Outcome Scale). In the intent-to-treat analysis, the SENTIS results did not achieve statistical significance for the primary efficacy end point: (OR, 1.17; 95\% CI, 0.81-1.67; $P=.407)$. The primary safety end point was met: There was no difference in serious adverse events between groups $(P=.923)$. A statistical trend for all-cause mortality and positive secondary safety end point outcomes was observed. ${ }^{1}$ Additionally, post hoc analyses have shown positive results, especially with regard to stroke-related mortality. In this report, we present further analyses of the mortality and severe disability data from the SENTIS trial.

\section{MATERIALS AND METHODS}

For detailed methods of the trial, we refer to the original publication of the SENTIS trial (ClinicalTrials.gov, No. NCT00119717). ${ }^{1}$ The trial was funded by CoAxia. All authors vouch for the accuracy and completeness of the data and analysis. All authors had access to all the data in the study and had final responsibility for submission of this publication.

Briefly, patients who met all of the enrollment criteria and provided written consent were allocated to NeuroFlo treatment with standard medical management (treatment) or standard medical management alone (control) through the use of a 1:1 randomization scheme that was stratified by site, baseline NIHSSS, and the time from symptom onset. All patients were followed for safety and efficacy through 90 days; formal clinical assessments were performed at 6 and 24 hours and at 4 (or hospital discharge, if earlier), 30, and 90 days. The final 90-day assessment was blinded. Follow-up cranial CT imaging was performed at 24 hours and, in cases of neurologic worsening, at any time up to 90 days. Additionally, a poststudy survey was completed to gather information on patient treatment unit allocation (admission to neuro/stroke intensive care unit, other intensive care unit, stroke unit, or general unit) and the presence of "do not resuscitate"/"do not intubate," and "comfort care only" orders.

\section{Statistical Analyses}

All analyses were performed on the "modified as-treated" population. ${ }^{1}$ Standard summary statistics were calculated for all study variables. Categoric variables were summarized by the use of frequency distributions and 95\% CIs.

The proportions of patients with all-cause and stroke-related mortality were compared between groups by use of a CochranMantel-Haenszel test, stratified by age and baseline NIHSSS. Logistic regression models were also fit for all-cause and strokerelated mortality to obtain an OR and 95\% CI for the treated versus not-treated comparison. Kaplan-Meier curves for stroke-related mortality were provided, along with estimates at specific time points $(4,30,60$, and 90 days $)$ and their associated 95\% CIs.

We qualitatively reviewed the serious adverse events related to deaths categorized into neurologic versus non-neurologic events and performed multiple logistic regression analyses for influence of baseline stroke severity (NIHSSS) and allocation of treatment location/unit.
We analyzed the severe disability and mortality data by dichotomized (mRS $0-4$ versus 5), tetrachotomized (mRS 0-2, 3-4, 5, 6 ), and full-scale (mRS 0, 1, 2, 3, 4, 5, 6) outcomes of the mRS.

All statistical tests, including those comparing the treatment groups for mortality, stroke-related mortality, and allocation to treatment location, are 2-sided. Statistical analyses were conducted in SAS version 9.1 or above (SAS Institute, Cary, North Carolina).

\section{RESULTS}

Between October 2005 and January 2010, 515 patients were enrolled in the SENTIS trial at 68 centers. A total of 257 patients were randomly assigned to the control group and 258 patients were randomly assigned to the treatment group (intention-to-treat population). Twenty-eight patients randomly assigned to treatment were excluded because of prespecified criteria, 5 patients randomly assigned to treatment did not receive treatment, and 1 patient randomly assigned to the control group received NeuroFlo treatment (both were protocol deviations), resulting in 261 not-treated patients and 226 treated patients in the "modified as-treated" analysis. ${ }^{1}$

\section{Mortality}

All-cause mortality rates (intention to treat: $11.2 \%$ versus $16.3 \%$; OR, 1.60; 95\% CI, 0.91-2.83; $P=.086$; modified intention to treat: $11.3 \%$ versus $16.3 \%$; OR, 1.56 ; $95 \% \mathrm{CI}, 0.87-2.80 ; P=$ .087 ), and stroke-related mortality (intention to treat: $7.8 \%$ versus 14.4\%; OR, 2.45; 95\% CI, 1.25-4.79; $P=.009$; modified intention to treat: $7.4 \%$ versus $14.4 \%$; OR, 2.53 ; $95 \%$ CI, $1.25-5.09$; $P=.010)$ rates did not differ between the intention to treat, modified intention to treat, and the from here on presented "modified as-treated" populations in treatment versus control patients.

As reported in the primary results, all-cause mortality ("modified as-treated") trended in favor of treated patients $(P=.079)$; by the 90 -day follow-up visit, $11.5 \%(26 / 226)$ in the treated group and $16.1 \%(42 / 261)$ in the not-treated group had died. ${ }^{1}$ Strokerelated mortality differed significantly between groups, again in favor of treated patients: $7.5 \%(17 / 226)$ treated versus $14.2 \%$ (37/ 261 ) not treated $(95 \% \mathrm{CI}-12.1,-1.2 ; P=.009)$. Most ( $85 \%)$ of the stroke-related deaths occurred within 30 days of the index stroke. The logistic regression analysis for freedom from strokerelated mortality, adjusted by baseline NIHSSS and age, favored treatment, with an OR of 2.41 (95\% CI, 1.22, 4.77; $P=.012$ ). Fig 1 shows the Kaplan-Meier curve for freedom from stroke-related mortality.

\section{Primary Causes of Stroke-Related Deaths}

For a full listing and definitions of stroke-related versus not stroke-related mortality, see Table 4 of the original SENTIS publication. ${ }^{1}$ Although the absolute numbers for each cause of strokerelated death were too few for any differences between arms to reach statistical significance, treated patients numerically had fewer stroke-related deaths for all causes except renal causes. Most notably, stroke-related deaths from neurologic causes (eg, bleeding, stroke progression, edema, new strokes) were numerically fewer in treated versus not-treated patients (treated: 9/17, 52.9\%; nontreated: $27 / 37,73.0 \% ; P=.214)$ as opposed to other reasons for death (eg, cardiac, renal, pulmonary, multi-organ failure, sepsis). 


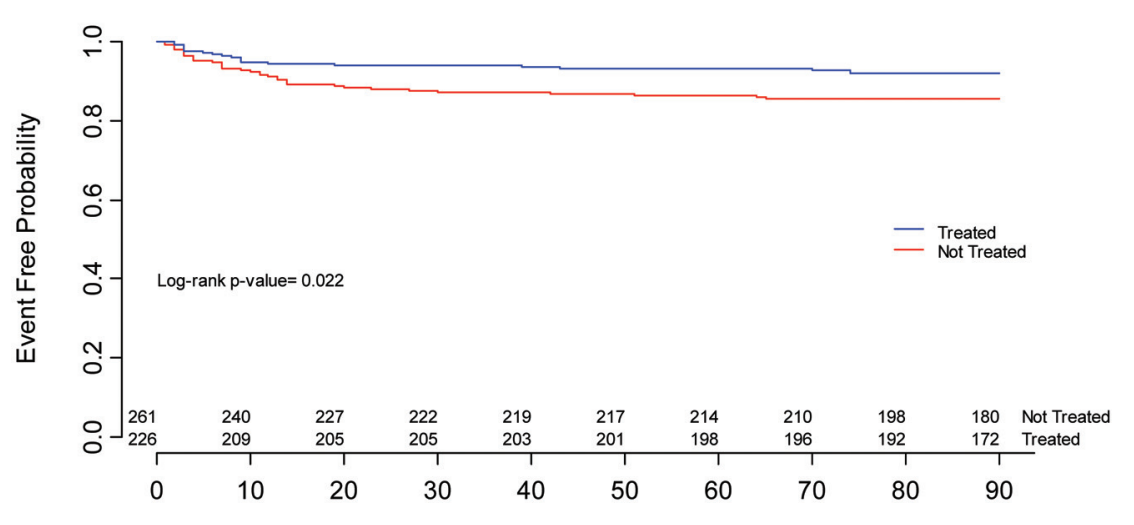

Days from Baseline

\begin{tabular}{|l|c|c|c|c|c|c|}
\hline & \multicolumn{3}{|c|}{$\begin{array}{c}\text { Treated } \\
\mathrm{N}=226\end{array}$} & \multicolumn{3}{c|}{$\begin{array}{c}\text { Not-treated } \\
\text { N=261 }\end{array}$} \\
\hline Time-point & Events (N) & $\begin{array}{c}\text { Cumulative } \\
\text { Events (N) }\end{array}$ & $\begin{array}{c}\text { Event Free Rate (\%) } \\
{[95 \% \text { Cl] }}\end{array}$ & Events (N) & $\begin{array}{c}\text { Cumulative } \\
\text { Events (N) }\end{array}$ & $\begin{array}{c}\text { Event Free Rate (\%) } \\
{[95 \% \text { Cl] }}\end{array}$ \\
\hline 4-Days & 5 & 5 & $97.8(94.7-99.1)$ & 12 & 12 & $95.4(92.0-97.4)$ \\
\hline 30-Days & 8 & 13 & $94.1(90.1-96.5)$ & 21 & 33 & $87.2(82.5-90.8)$ \\
\hline 60-Days & 2 & 15 & $93.2(89.0-95.8)$ & 2 & 35 & $86.4(81.6-90.1)$ \\
\hline 90-Days & 2 & 17 & $92.2(87.8-95.1)$ & 2 & 37 & $85.6(80.7-89.4)$ \\
\hline
\end{tabular}

The Greenwood's formula was used to calculate the $95 \%$ confidence interval for the Kaplan-Meier estimates for survival for each randomization group.

FIG 1. Kaplan-Meier curve: Freedom from stroke-related mortality.

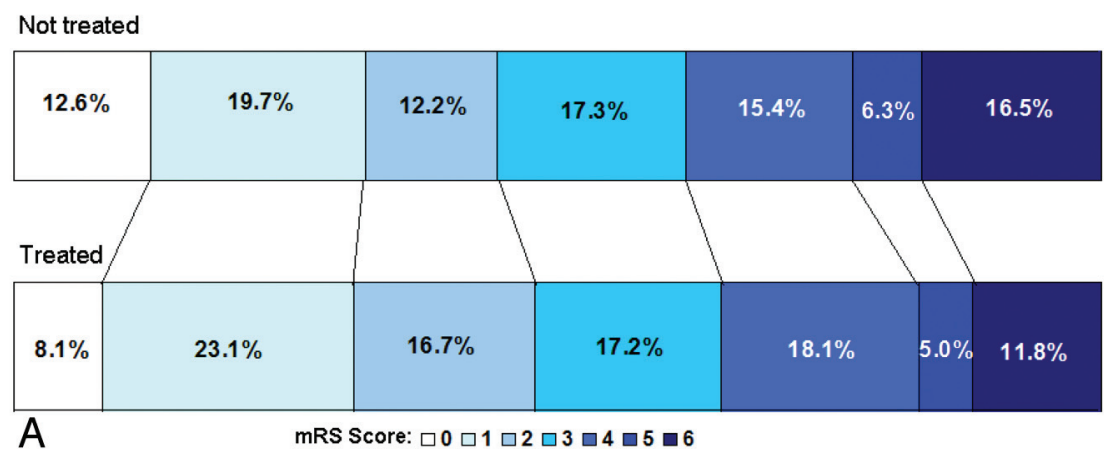

Not treated

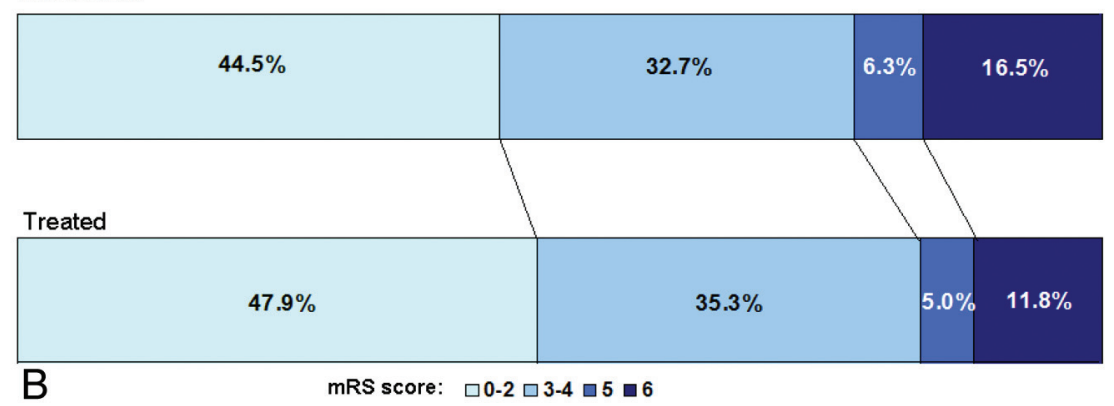

FIG 2. Modified Rankin shift for SENTIS as-treated population. *Values within the shift analysis are based on 90-day evaluable patients.

\section{Severe Disability}

The trend toward reduced all-cause mortality, mainly driven by a significantly reduced stroke-related mortality, did not come at the cost of an increased rate of severe disability. Among the survivors at the 90-day follow-up, fewer of the treated $(5.6 \%, 11 / 195)$ patients than the control patients $(7.5 \%, 16 / 212)$ were severely disabled ( $\mathrm{mRS}$ score $=5)$. The absolute difference $(1.9 \%)$ was not statistically significant in either univariate or multivariate analyses (OR, 0.58; 95\% CI, $0.24,1.39 ; P=.223$ ). As shown in Fig 2, compared with the not-treated group, the treated group had more patients in the lower range of the mRS scores and fewer with scores that indicate death (mRS 6) and severe disability (mRS 5). This suggests that after NeuroFlo treatment, patients who may have otherwise died (mRS 6) or been severely disabled (mRS 5) were more likely to survive with moderate or mild disability. Additionally, patients who may have been moderately disabled (mRS 3-4) were shifted toward an independent outcome (mRS $0-2)$.

\section{First Site of Care}

A total of 437 of 515 (85\%) poststudy surveys were returned with evaluable data regarding first site of care; 204 of 226 for treated patients and 233 of 261 for control patients. The survey suggested differences in the first site of care between treatment arms with nominally more treated patients admitted before the procedure to a stroke- or neuro-intensive care unit than not-treated patients (Table 1). When all intensive care units were combined, the numbers were significant, with an absolute difference of $13.5 \%(60.3 \%$ versus $46.8 \%, P=$ .005). Conversely, more patients from the not-treated arm were admitted to general wards as their first site of care. When these variables were included in the multivariate analysis, first site of care was not a predictor of mortality, strokerelated mortality, or independent outcome categorized as mRS $0-2$ versus 3-6. Furthermore, when the outcome of all-cause mortality is analyzed by the first site of care, the treated group had lower rates of mortality $(8.5-23.1 \%)$ than the not-treated group (14.4$20.6 \%$ ) for all sites of care except for general unit (treated, $23.1 \%$ versus not treated, 20.6\%; $P=1.000$ ) (Table 2). Similarly, the stroke-related mortality rate was also nominally lower in the treated group (7.3-15.4\%) compared with the not-treated group (11.1-17.7\%) for all first sites of care except general unit (treated, $15.4 \%$ versus not treated, 
Table 1: First site of care

\begin{tabular}{|c|c|c|c|}
\hline \multirow[b]{3}{*}{ First Site of Care } & Treated & Not Treated & \multirow{3}{*}{$\begin{array}{c}P \text { Value } \\
\text { (Fisher Exact) }\end{array}$} \\
\hline & $N=204$ & $N=233$ & \\
\hline & $n=25$ & $n=38$ & \\
\hline Neuro/Stroke ICU & $40.2 \%(82 / 204)$ & $32.2 \%(75 / 233)$ & .090 \\
\hline Other ICU & $20.1 \%(41 / 204)$ & $14.6 \%(34 / 233)$ & .162 \\
\hline Stroke unit & $33.3 \%(68 / 204)$ & $38.6 \%(90 / 233)$ & .273 \\
\hline General unit & $6.4 \%(13 / 204)$ & $14.6 \%(34 / 233)$ & .008 \\
\hline Any ICU & $60.3 \%(123 / 204)$ & $46.8 \%(109 / 233)$ & .005 \\
\hline
\end{tabular}

Note:-Nindicates total number of surveys received with evaluable data for first site of care; $n$, number of patients with evaluable surveys who died.

${ }^{a}$ Any ICU is the combination of the Neuro/Stroke ICU and Other ICU.

Table 2: All-cause mortality rate by first site of care

\begin{tabular}{|c|c|c|c|}
\hline \multirow[b]{3}{*}{ First Site of Care } & Treated & Not Treated & \multirow{3}{*}{$\begin{array}{c}P \text { Value } \\
\text { (Fisher Exact) }\end{array}$} \\
\hline & $N=204$ & $N=233$ & \\
\hline & $n=25$ & $n=38$ & \\
\hline Neuro/Stroke ICU & $8.5 \%(7 / 82)$ & $16.0 \%(12 / 75)$ & .220 \\
\hline Other ICU & $14.6 \%(6 / 41)$ & $17.7 \%(6 / 34)$ & .760 \\
\hline Stroke unit & $13.2 \%(9 / 68)$ & $14.4 \%(13 / 90)$ & 1.000 \\
\hline General unit & $23.1 \%(3 / 13)$ & $20.6 \%(7 / 34)$ & 1.000 \\
\hline Total mortality rate & $12.3 \%$ & $16.3 \%$ & .275 \\
\hline
\end{tabular}

Note: - N indicates total number of surveys received with evaluable data for first site of care; $n$, number of patients with evaluable surveys who died.

Table 3: Stroke-related mortality rate by first site of care

\begin{tabular}{|c|c|c|c|}
\hline \multirow[b]{3}{*}{ First Site of Care } & Treated & Not Treated & \multirow{3}{*}{$\begin{array}{c}P \text { Value } \\
\text { (Fisher Exact) }\end{array}$} \\
\hline & $N=204$ & $N=233$ & \\
\hline & $n=17$ & $n=33$ & \\
\hline Neuro/Stroke ICU & $7.3 \%(6 / 82)$ & $16.0 \%(12 / 75)$ & .131 \\
\hline Other ICU & $7.3 \%(3 / 41)$ & $17.7 \%(6 / 34)$ & .285 \\
\hline Stroke unit & $8.8 \%(6 / 68)$ & $11.1 \%(10 / 90)$ & .792 \\
\hline General unit & $15.4 \%(2 / 13)$ & $14.7 \%(5 / 34)$ & 1.000 \\
\hline $\begin{array}{c}\text { Total stroke-related } \\
\text { mortality rate }\end{array}$ & $8.3 \%$ & $14.2 \%$ & .070 \\
\hline
\end{tabular}

Note:-N indicates total number of surveys received with evaluable data for first site of care; $n$, number of patients with evaluable surveys who died of stroke-related causes.

$14.7 \% ; P=1.000$ ) (Table 3). Therefore, the differences in allocation of care do not account for the reduced mortality rates among the treated patients.

\section{Do Not Resuscitate/Do Not Intubate and Withdrawal of Care Orders}

The numbers for do not resuscitate/intubate, and comfort care orders did not differ between treatment and control patients: $10.3 \%(21 / 204)$ versus $11.7 \%(28 / 239)$ (Fisher exact test, $P=$ $.652)$, and $6.9 \%(14 / 204)$ versus $10.5 \%(25 / 239)(P=.239)$. Death was highly associated with the presence of these orders (OR, 22.96; 95\% CI, 8.5, 62.3; $P<.0001$ ) and comfort care orders (OR, 77.33; 95\% CI, 23.3, 218.5; $P<.0001)$. The median number of days between initiation of the orders and death was 2.0 days for do not resuscitate/intubate orders and 2.5 days for comfort care orders; there was no difference between treated and not-treated patients.

\section{DISCUSSION}

The SENTIS trial tested the clinical efficacy and safety of the NeuroFlo device, which, by increasing cerebral blood flow to ischemic brain, was hypothesized to lead to reduced morbidity and mortality in patients with acute stroke treated within 14 hours after onset of symptom. ${ }^{1}$ SENTIS established safety for the NeuroFlo procedure but missed statistical significance for the primary clinical outcome end point. While the use of the global outcome end point and also dichotomized end points of the mRS have been a matter of recent debate, ${ }^{6-8}$ the currently favored mRS shift analysis over the full range of the ordinal scale did not render a different trial result. ${ }^{1}$ An intriguing and important observation in the SENTIS trial was a trend for reduced overall mortality. This reduction was driven by a significantly lower stroke-related mortality rate among treated patients. Death rates from stroke, strokerelated complications, and new strokes were all nominally lower in the treated patients. Notably, the reduction in the death rate was not accompanied by an increase in the proportion of patients who were severely disabled. Additionally, these findings were not related to the first site of care or withdrawal of care in treated versus not-treated patients.

Albeit dealing with post hoc analyses, these results shed more light on the findings from SENTIS. It is apparent that the trend in reduced all-cause mortality was for the most part a result of reduced stroke-related mortality. Hemodynamic augmentation by partial aortic occlusion results in a varying increase of CBF by approximately $30 \%$, an effect that lasts beyond the procedure itself. ${ }^{5}$ Although further analysis of the acquired imaging data are necessary, this may lead to improved collateral flow to ischemic penumbral brain and thereby reduce infarct size in treated patients as compared with control subjects. Stroke size has been repeatedly established as a predictor for outcome and mortality. ${ }^{9-12}$ Both direct stroke-related complications (eg, space-occupying infarction with increased intracranial pressure, edema) and secondary disability-associated complications (eg, deep venous thrombosis, infections, aspiration) may be reduced as a result of a smaller final infarct size. Although SENTIS was not powered to detect the effect on overall clinical outcome measured with the mRS, the mortality findings are encouraging and may be useful in designing further studies. Some of the promising approaches with other acute stroke treatments that have been studied in pilot trials are the use of partial aortic occlusion as an adjunct to standard rtPA (FastFlo) ${ }^{3}$ and in extended time windows (Flo24). ${ }^{4}$ In both trials, feasibility and safety could be demonstrated. A larger NeuroFlo device with a central lumen has been developed, which could allow for intraarterial treatment and/or thrombectomy in parallel to partial aortic occlusion and flow improvement. The latter has not been tested in a pilot trial.

A frequently disputed ethical as well as economic issue is whether a therapeutic procedure reduces mortality at the cost of increasing severe disability in survivors. ${ }^{13}$ In SENTIS, the reduction in mortality did not result in an increase in severe disability as measured by the mRS. This is not explained by a difference in the rate of serious adverse advents between the 2 arms and illustrates an overall shift toward better outcomes in the treatment arm, an effect also observed in the hemicraniectomy trials. ${ }^{14}$

Potential confounding variables in assessing mortality outcomes in stroke trials are variability in admission to intensive care units and stroke units and variability in the application of do not resuscitate or intubate orders and compassionate care, a frequent phenomenon especially among victims of ischemic stroke and 
hemorrhagic stroke. ${ }^{15}$ It is theoretically possible, in interventional trials unblinded for the treatment arm and only blinded for the outcome assessment (PROBE design), that actively treated patients may more frequently receive specialized care and allocation to intensive care and stroke units, leading to self-fulfilling results. In a poststudy survey, we acquired further data regarding site of care and do not resuscitate/intubate orders not originally obtained for the SENTIS trial itself. There were no major differences with regard to treatment allocation or do not resuscitate/intubate orders; however, more patients treated with NeuroFlo were assigned to stroke units and neuro-intensive care units. This difference in first site of care may be due in part to standard of care procedures and hospital protocols that often require intensive care unit admission after any endovascular procedure. Despite the allocation imbalance, in treated patients, both the allcause and stroke-related mortality rates were nominally lower in all but one allocation (general ward), where they were nearly identical. Finally, first treatment site was not an independent predictor of outcome in the multivariate analysis.

Among the limitations of our analyses are the inclusion of post hoc analyses and the use of a poststudy survey, though the latter was submitted for $85 \%$ of the study patients. Despite these limitations, we believe that the significant reduction in stroke-related mortality in the SENTIS trial is an indication of potential efficacy of the therapeutic approach. We did not find other explanations for this observation and therefore do not believe this to arise from confounds in sites of or intensity of care, though SENTIS was ultimately not powered for the predefined end point. We believe that the therapeutic approach of hemodynamic augmentation merits further study because the efficacy results are promising and safety is established.

\section{ACKNOWLEDGMENTS}

The authors would like to thank the following: Lisa M. Thackeray, MS, of The Integra Group for statistical support; and Kay Zander, MA, and Ellen O'Malley, MS, of CoAxia, Inc, for valuable input, review, and editing of the manuscript.

Disclosures: Peter D. Schellinger-RELATED: Consulting Fee or Honorarium: CoAxia; Support for Travel to Meetings for the Study or Other Purposes: CoAxia; Fees for Participation in Review Activities, Such as Data Monitoring Boards, Statistical Analysis, Endpoint Committees, and the Like: CoAxia; UNRELATED: Board Membership: Boehringer Ingelheim, Cerevast; Consultancy: Boehringer Ingelheim, Cerevast; Payment for Lectures (including service on speakers bureaus): Boehringer Ingelheim, Cerevast. Ashfaq Shuaib_RELATED: Grant: CoAxia*; Consulting Fee or Honorarium: CoAxia; Support for Travel to Meetings for the Study or Other Purposes: CoAxia; UNRELATED: Consultancy: Pfizer, BI, Sanofi, Bayer, AstraZeneca, Lundbeck, dPharm, Comments: Consultancy/advisory boards; Grants/Grants Pending: Lundbeck, ${ }^{*}$ dPharm, ${ }^{*}$ Asubio, ${ }^{\star}$ Photothera, ${ }^{*}$ Brainsgate*; Payment for Lectures (including service on speakers bureaus): AstraZeneca, Bayer, Bl, Sanofi, Pfizer, Merck, dPharm, Asubio. Martin Köhrmann—RELATED: Consulting Fee or Honorarium: CoAxia; Support for Travel to Meetings for the Study or Other Purposes: CoAxia*; UNRELATED: Board Membership: Boehringer Ingelheim; Payment for Lectures (including service on speakers bureaus): Boehringer Ingelheim, Bayer Healthcare, Novartis. David Liebeskind-RELATED: Grant: NIH, ${ }^{*}$ Comments: P50NS044378, K24NS072272, R13NS082049. Maxim Hammer-UNRELATED: Expert Testimony: Roetzel and Andress (a legal professional organization), Comments: I have provided medical expert testimony on stroke cases for legal firms. Souvik Sen-RELATED: Consulting Fee or Honorarium: Consultant fee from CoAxia, Inc (2 years ago); UNRELATED: Grants/Grants Pending: National Institutes of Health*; Payment for Lectures (including service on speakers bureaus): Speakers bureau for Boehringer Ingelheim, Genentech, Sanofi-Synthélabo, and Bristol-Myers Squibb. Rishi GuptaRELATED: Consulting Fee or Honorarium: CoAxia, Comments: less than $\$ 5000$ over
24 months; UNRELATED: Consultancy: Stryker Neurovascular (less than $\$ 10,000$ over 12 months), Covidien (less than $\$ 10,000$ over 12 months); OTHER RELATIONSHIPS: Associate Editor, Journal of Neuroimaging. Jeffrey L. Saver-RELATED: Grant: CoAxia*; Consulting Fee or Honorarium: CoAxia*; Support for Travel to Meetings for the Study or Other Purposes: CoAxia*; UNRELATED: Consultancy: Grifols, ${ }^{*}$ Covidien, ${ }^{*}$ Lundbeck*; Grants/Grants Pending: NIH-NINDS*; OTHER RELATIONSHIPS: My employer, the University of California, has some patent rights on retrieval devices for stroke. I am not a party or beneficiary to the arrangements ( ${ }^{*}$ money paid to institution). CoAxia, Inc, provided funding for the SENTIS Trial. Tudor Jovin, MD: TJ is employed by the University of Pittsburgh, Pittsburgh, Pennsylvania. The University received clinical trial payments based on the number of patients enrolled in the SENTIS trial from CoAxia. He has received honorarium from CoAxia for consulting work with the company; David Y. Huang, MD: DYH is employed by the University of North Carolina, Chapel Hill, North Carolina. The University received clinical trial payments based on the number of patients enrolled in the SENTIS trial from CoAxia. Sten Solander, MD: SS is employed by the University of North Carolina, Chapel Hill, North Carolina. The University received clinical trial payments based on the number of patients enrolled in the SENTIS trial from CoAxia. He has received consulting fees for consulting from CoAxia. Ronen R. Leker, MD: RRL is employed by the HadassahHebrew University Medical Center, Jerusalem, Israel. Hadassah received clinical trial payments based on the number of patients enrolled in the SENTIS trial from CoAxia.

\section{REFERENCES}

1. Shuaib A, Bornstein NM, Diener HC, et al. Partial aortic occlusion for cerebral perfusion augmentation: Safety and Efficacy of NeuroFlo in Acute Ischemic Stroke trial. Stroke 2011;42:1680-90

2. Hammer M, Jovin T, Wahr JA, et al. Partial occlusion of the descending aorta increases cerebral blood flow in a nonstroke porcine model. Cerebrovasc Dis 2009;28:406-10

3. Emery DJ, Schellinger PD, Selchen D, et al. Safety and feasibility of collateral blood flow augmentation after intravenous thrombolysis. Stroke 2011;42:1135-37

4. Hammer MD, Schwamm L, Starkman S, et al. Safety and feasibility of NeuroFlo use in eight- to 24-hour ischemic stroke patients. Int J Stroke 2012;7:655-61

5. Liebeskind DS. Aortic occlusion for cerebral ischemia: from theory to practice. Curr Cardiol Rep 2008;10:31-36

6. Schellinger PD, Bath PM, Lees KR, et al. Assessment of additional endpoints for trials in acute stroke: what, when, where, in who? Int J Stroke 2012;7:227-30

7. Lees KR, Bath PM, Schellinger PD, et al. Contemporary outcome measures in acute stroke research: choice of primary outcome measure. Stroke 2012;43:1163-70

8. Bath PM, Lees KR, Schellinger PD, et al. Statistical analysis of the primary outcome in acute stroke trials. Stroke 2012;43:1171-78

9. Davis SM, Donnan GA, Parsons MW, et al. Effects of alteplase beyond $3 \mathrm{~h}$ after stroke in the Echoplanar Imaging Thrombolytic Evaluation Trial (EPITHET): a placebo-controlled randomised trial. Lancet Neurol 2008;7:299-309

10. Thomalla GJ, Kucinski T, Schoder V, et al. Prediction of malignant middle cerebral artery infarction by early perfusion- and diffusionweighted magnetic resonance imaging. Stroke 2003;34:1892-99

11. Albers GW, Thijs VN, Wechsler L, et al. Magnetic resonance imaging profiles predict clinical response to early reperfusion: the diffusion and perfusion imaging evaluation for understanding stroke evolution (DEFUSE) study. Ann Neurol 2006;60:508-17

12. Mlynash M, Lansberg MG, De Silva DA, et al. Refining the definition of the malignant profile: insights from the DEFUSE-EPITHET pooled data set. Stroke 2011;42:1270-75

13. Juttler E, Schellinger PD, Aschoff A, et al. Clinical review: therapy for refractory intracranial hypertension in ischaemic stroke. Crit Care 2007;11:231

14. Vahedi K, Hofmeijer J, Juettler E, et al. Early decompressive surgery in malignant infarction of the middle cerebral artery: a pooled analysis of three randomised controlled trials. Lancet Neurol 2007;6:215-22

15. Steiner T, Mendoza G, De Georgia M, et al. Prognosis of stroke patients requiring mechanical ventilation in a neurological critical care unit. Stroke 1997;28:711-15 